\title{
CRISPR/Cas Class 2 systems and their applications in biotechnological processes
}

\author{
D.S. Mota ${ }^{1}$, J.M. Marques ${ }^{1}$, J.M. Guimarães ${ }^{1}$ and L.A.M. Mariúba ${ }^{2}$ \\ ${ }^{1}$ Programa de Pós-Graduação em Biotecnologia, Universidade Federal do \\ Amazonas, Manaus, AM, Brasil \\ ${ }^{2}$ Núcleo de Inovação Tecnológica, Instituto Leônidas \& Maria Deane - \\ Fiocruz Amazônia, Manaus, AM, Brasil
}

Corresponding author: L.A.M. Mariúba

E-mail: lamariuba@hotmail.com

Genet. Mol. Res. 19 (1): gmr18478

Received August 21, 2019

Accepted March 11, 2020

Published March 31, 2020

DOI http://dx.doi.org/10.4238/gmr18478

\begin{abstract}
Since 2012, CRISPR/Cas technologies have revolutionized the field of modern molecular biology and biotechnology due to their ability to direct a Cas nuclease to cleave specific nucleic acid sequences with a guide RNA (crRNA), providing dynamic tools for many applications, being naturally derived from the adaptive immune system of prokaryotes. The most widely used toolbox for genome editing, modulation, and detection contains types II, V and VI of class 2 systems, categorized and characterized by Cas9, Cas12, and Cas13, respectively. In recent years, given the wide application of Class 2 CRISPR-Cas systems as molecular tools, there has been a focus on the discovery, characterization, and specificity of Cas effector nucleases from these systems, generating even more possibilities for the development of new applications. We reviewed the scientific literature on current knowledge of CRISPR-Cas systems and recent advances in biotechnology involving class 2 systems between 2000 and 2019. For this, we searched for original articles indexed in the following databases: PubMed, Scopus, SciELO, Periodicos Capes and Google Scholar, and used keyword combinations such as "CRISPR / Cas Class 2 systems", "Cas9", "Cas12", "Cas13", "Genome editing" and "diagnostic detection". Eighty original articles were obtained, 55 of
\end{abstract}


them were selected for this review because they match with the keywords and the years of publication.

Key words: Gene editing; RNA cleavage; Biotechnological tools

\section{INTRODUCTION}

Bacteria and archaea domains have developed different defense mechanisms against foreign nucleic acids derived from bacteriophages and mobile genetic elements (MGEs), among them, clustered regularly interspaced short palindromic repeats (CRISPRs) together with "CRISPR associated proteins" (CAS) work together to form an adaptive immune system called "CRISPR/Cas". The system consists of several partially palindromic conserved repeats separated by small, unique DNA patches, called spacers. They are acquired from invading genetic elements, thus generating a "molecular memory" for further targeting of a foreign nucleic acid using base complementarity of the coded sequence to effect the cleavage (Barrangou et al., 2007; Garneau et al., 2010). CRISPR/Cas systems are currently grouped into two distinct classes, six types and more than 30 subtypes that differ in their components and mechanisms of action. The determining feature between two classes is the effector nature of cleaving the target sequence; the Class 1 contains Cas protein complexes with multiple subunits, while Class 2 is characterized by having only one effector protein. The nature of Class 2 systems allows them to be widely employed in a biotechnological toolkit since there are fewer components to use compared to Class 1 systems (Koonin et al., 2017). Over the past decade, the ability to reprogram Cas proteins by creating crRNAs (guide RNAs) designed to bind and/or cleave specific DNA or RNA sequences has made them useful in a variety of applications, including genome editing and modulation, specific control of gene expression and nucleic acid detection (Wang et al., 2016). The expansion in knowledge about CRISPR/Cas systems has motivated the development of various research tools and has made a major impact in virtually all areas of life sciences, supporting key applications in various areas such as biotechnology, agriculture and medicine. The focus of this review is to provide a brief description of the current knowledge of CRISPR/Cas system, describing its discovery, function, and classification, especially concerning recent advances in the application of this powerful tool in biotechnological research using Class 2. This review provides information about the CRISPR/Cas system as well as the different class 2-based biotechnological applications. Bibliographic sources were consulted to identify original studies developed with CRISPR/Cas system, published in databases such as PubMed, Scopus, SciELO, "Periodicos Capes", and Google Scholar using the following keywords: CRISPR/Cas systems; CRISPR/Cas Class 2 systems; Cas9; Cas12; Cas13; Genome editing and diagnostic detection. As criteria for inclusion, consulted scientific articles were published from 2000 to 2019.

\section{CRISPR/CAS SYSTEM DISCOVERY}

CRISPR system was first reported in 1987, when Ishino et al. (1987) observed the presence of a genetic structure containing five homologous repeats of 29 nucleotides being interrupted by 32 non-repetitive nucleotide sequences (spacers) in E. coli during an analysis 
of gene sequences involved in phosphate metabolism. This was followed by numerous reports of similar sequences in other microbes; however, until the mid-2000s there was no clear evidence of the biological function or mechanism of these sequences (Van Soolingen et al., 1993). In 2002, the acronym CRISPR/Cas was officially named by Jansen et al. (2002) to bring uniformity to the description of this sequences. In addition, the discovery of a conserved set of operonorganized Cas genes located adjacent to a CRISPR locus, indicating the Cas genes along with the CRISPR locus have a functional relationship in prokaryotic organisms (Van Der Oost et al., 2009). The involvement of CRISPR elements as a hereditary adaptive immune system was experimentally confirmed in 2005 when Bolotin et al. (2005), Mojica et al. (2005) and Pourcel et al. (2005) independently reported in their research that spacers were similar to sequences derived from invasive mobile genetic elements (MGEs), including bacteriophages, plasmids and transposons. Indeed, comparative genomic analyzes have suggested that the CRISPR system and Cas proteins actually work together and constitute an acquired immunity system to protect prokaryotic cells against invading DNAs, similar to eukaryotic RNA interference system (RNAi) (Mojica et al. 2016). In 2012 a major milestone in CRISPR technology revolutionized the field of molecular biology and genetic engineering, the group led by Doudna and Charpentier published an article revealing the first use of CRISPR with Cas9 endonuclease being programmed with simple RNA molecules to cleave in vitro specific sites in DNA strands (Jinek et al., 2012). Since this pioneering and subsequent works, the CRISPR / Cas system has gained wide acceptance over other systems due to its simplicity, speed and efficiency in modifying genes in any particular cell or tissue (Du et al., 2016).

\section{CRISPR BIOLOGICAL MECHANISM: THE THREE PHASES OF ADAPTIVE IMMUNITY}

At the molecular level, CRISPR/Cas-mediated immunity works through three main steps: spacer adaptation or acquisition; crRNA processing or maturation and target interference or cleavage (Figure 1).

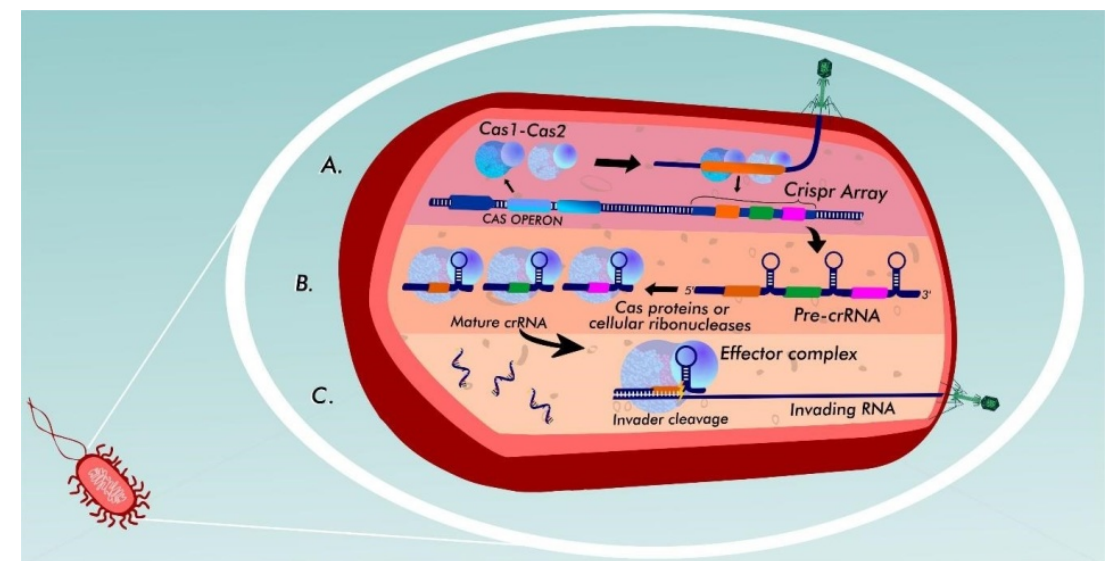

Figure 1. CRISPR/Cas immune adaptive process. The CRISPR-Cas defense mechanism occurs in three stages: adaptation, maturation and interference. (A) Adaptation: Invasive nucleic acid is recognized by the Cas1-Cas2 complex, incorporating the invasive sequence into the CRISPR spacer region. (B) Maturation: Pre-crRNA is generated by transcription of the CRISPR region and is processed into smaller RNA units, creating individual mature crRNAs. (C) Interference: Mature crRNAs guide Cas nucleases to specifically target their respective target nucleic acid sequences, resulting in cleavage of foreign DNA or RNA. 
The adaptation or acquisition of spacers is the first stage in the construction of prokaryotic cell genetic memory. During this stage, Cas1 and Cas2 are the major proteins that plays crucial roles in recognizing and acquiring new protospacer DNA fragments as new spacer sequences in the CRISPR matrix during the first exposure to foreign nucleic acids (Figure 1A) (Yosef et al., 2012).

The second stage is crRNA processing, when after a second infection the components for target nucleic acid cleavage are synthesized to form the RNA-guided nuclease complex. Transcription of the CRISPR array begins with a primary RNA (precrRNA) within the leader region which is subsequently processed into a set of mature smaller RNAs (crRNA) which guide Cas proteins to invasive target sequences (Figure 1B) (Deltcheva et al., 2011). Interference is the final stage of CRISPR adaptive immunity, which results in the cleavage of foreign nucleic acid by Cas nuclease through proper base pairing between the crRNA with the complementary sequence of double stranded DNA or single stranded RNA (Figure 1C). Thus, the processing and interference steps would be similar to the immune response of a "vaccinated" host against a particular specific nucleic acid (Marraffini et al., 2010).

\section{CRISPR/CAS SYSTEM DIVERSITY AND CLASSIFICATION}

CRISPR/Cas systems exhibit remarkable diversity and are present in almost all archaea, and in approximately $50 \%$ of analyzed bacteria domain, being absent in eukaryotes and viruses (Makarova et al., 2015).

These systems are classified into two general classes based on phylogenetic analysis, comparative genomics and protein constitution. Class 1 systems consist of a complex machinery, with multiple Cas proteins assisting in the recognition and cleavage of foreign nucleic acids, while in the Class 2 system, they are formed from a single interference domain Cas effector protein. Interestingly Class 2 systems have only $10 \%$ of all sequenced CRISPR/Cas loci in prokaryote genomes, almost all within the bacteria domain (Wiedenheft et al., 2011).

CRISPR systems have been further classified into six types and grouped into two classes: types I, III and IV (Class 1) and types II, V and VI (Class 2), which are currently subdivided into approximately 30 subtypes (Shmakov et al., 2015). Type I, II, and V (and probably IV) feature as target double stranded invading DNAs, while Type III systems cleave both DNA and RNA. Type VI systems exclusively cleave RNA, defending against RNA phages. In addition, systems which operate through DNA segmentation, each protospacer is made up of a short (2-5 bp) sequence called the protospacer adjacent motif (PAM), which plays a key role in immunologic recognition and spacer acquisition. In contrast, type III and VI complexes are regulated by sequences flanking protospacers of the target RNAs, referred as protospacer flanking site (PFS) (Koonin, 2017).

\section{BIOTECHNOLOGICAL APPLICATIONS USING CRISPR-CAS CLASS 2 GENES}

The molecular characteristics of CRISPR/Cas Class 2 systems are the most used among researchers due to their simplicity and mechanism, depending only on small RNA molecules and Cas-specific protein. Class 2 has provided the development of molecular 
toolkits for various purposes, initiated with type II and followed by types V and VI (Mohanraju et al., 2016).

\section{Type II: CRISPR/Cas9}

Cas9 endonuclease was the first CRISPR-associated effector protein to be widely used in genetic engineering. Cas 9 has a bilobulated form containing a recognition lobe (REC) and a nuclease lobe (NUC), while the REC lobe is composed of two targeting domains, REC1 and REC2, the NUC lobe has the RuvC, HNH and PI domains. The HNH and RuvC nuclease domains are responsible for cleavage of the complementary and noncomplementary DNA strands, respectively. The PI domain recognizes the adjacent protospacer motif (PAM) sequence in non-complementary chain. Mature crRNA associates with a trans-activating crRNA (tracrRNA) resulting in a tracrRNA-crRNA secondary structure that can direct Cas9 nuclease to induce DNA breaks in the PAM region (Nishimasu et al., 2014).

The CRISPR/Cas9 system has been used efficiently by the scientific community in different types of cells and organisms, including in therapeutic treatments of human diseases such as cancer, genetic disorders, viral diseases, as well as use in plants, bacteria and fungi.

- Oncology: The therapeutic uses of CRISPR/Cas9 system proved to be an extremely valuable and efficient tool for cancer genetic research. CRISPR/Cas9 has been used to accelerate the development of T cells chimeric antigen receptor (CAR) (Ren et al., 2017). It can also be used with pro-apoptotic effect on cancer cells by knocking out Programmed cell death protein 1 (PD-1) (Zhao et al., 2018). It can be used alone or in combination with other treatments for in vitro and in vivo studies to combat the etiological factors HPV16 and HPV18 of cervical cancer (Zhen et al., 2016);

- Genetic Diseases: For many genetic diseases CRISPR/Cas9 technology can be used due its ability to produce genome-specific changes. This includes diseases such as Duchenne muscular dystrophy (DMD) caused by mutations in a gene located on the $\mathrm{X}$ chromosome. In this experiment, authors used a mouse model which have DMD, and obtained a dystrophy gene mutation correction in its descendants, carrying $2-100 \%$ of the corrected gene (Egorova et al., 2019). In another research authors were also able to correct mutant intestinal stem cells of cystic fibrosis patients, and restore their function in vitro, providing the first step to advance gene therapy in CRISPR cystic fibrosis patients (Schwank et al., 2013). Clinical trial applications for the treatment of sickle cell disease using the CRISPR/Cas system have also made promising advances by correcting diseasecausing mutations in pluripotent stem cells with Cas9-mediated activity and no off-target effects ( $\mathrm{Li}$ et al., 2016a).

-Viral Diseases: CRISPR systems can be employed to combat viral diseases by disrupting viral replication mechanisms and restoring infected cells to normal. One of the most intensely investigated viral diseases is HIV, for which CRISPR/Cas9 was widespread in various therapeutic approaches. Kaminski et al. (2016) for example, employed Cas9/crRNA machinery for precisely remove excision of the HVI-1 provirus, being administered in culture of latently infected human CD4 + T cells. Recently, Wang et al. (2018) demonstrated the SaCas9/sgRNA gene-editing complex could be programmed to excise the latent HIV-1 provirus and therefore inhibit HVI-1 infection. In addition, Cas9 has 
been used to target conserved regions, which are responsible for the persistence and replication of hepatitis B virus (HBV) (Zeisel et al., 2015);

- Antimicrobial Potential: Misuse or overuse of antibiotics has led to an increasing number of antimicrobial resistant bacteria. CRISPR/Cas9 system can be used as alternative to use alongside conventional antibiotics or as programmable antibiotic to deactivate specific bacterial sequences, avoiding drug resistance or returning of bacterial toxicity by cleaving crucial domains in your genome without disturbing remaining microbiota (Bikard et al., 2014);

- Agricultural applications: The practicality provided by CRISPR/Cas9 technology in genetic editing has provided user-friendly tools for developing new plant varieties, improving agricultural production, conferring resistance to certain viral, fungal or bacterial diseases, and modifying characteristics as the fruit size. The system was employed, for example, to develop cucumbers with broad virus resistance without affecting plant development (Chandrasekaran et al., 2016) and to improve yield of maize crops under water stress (Shi et al., 2017). It has also been successfully applied to different fruit trees, including strawberry (Zhou et al., 2018), banana (Kaur et al., 2018), apple (Nishitani et al., 2016) and kiwi (Wang et al., 2018a).

\section{Type V: CRISPR / Cas12}

CRISPR-based genome editing tools have expanded by the discovery of new Class 2 effector proteins, such as type $\mathrm{V}$ proteins. Like Cas9 in size and shape, the Cas 12 effector protein consists into two main lobes: one encompasses a nuclease lobe (NUC) and another of alpha helical recognition (REC). The REC lobe comprises two domains (REC1 and REC2), while the NUC lobe is composed of the RuvC domains (subdivided into I, II and III), PI, WED and $\mathrm{BH}$, being absent the $\mathrm{HNH}$ domain, in contrast to the Cas9 endonuclease (Safari et al., 2019).

The Cas12 system does not require a tracrRNA for processing mature crRNAs, which are processed by ribonuclease activity of Cas 12 itself. In addition, Cas 12 generates sticky ends which could increase the insertion efficiency of new DNA sequences in contrast to blind ends created by Cas9 (Jeon et al., 2018). With these advantages, Cas12 system has unique features to extend new genome editing tools, which can be used in a variety of biological approaches, including base editing, multiplex gene segmentation and epigenetic modulation (Safari et al., 2019).

Based on enzymatic characteristics Cas12 system was used to edit genome in various organisms. Its function was initially tested in E. coli and obtained repression results with high degree of specificity and little off-target effect (Zhang et al., 2017). Similarly, the Cas12 system was used in Clostridium genus to produce a large number of chemicals and fuels, such as n-butanol (Li et al., 2016b). In addition Cas 12 has also been successfully used for genome editing in mammals, including human cells (Tsukamoto et al., 2018) mice (Teng et al., 2019) and swine (Wu et al., 2018).

The Cas 12 system also has promises for use in nucleic acid detection methods for human genotyping and pathogen detection. Chen et al. (2018) recently reported the application of a technique for diagnosing invasive nucleic acids called DETECTR (DNA Endonuclease Targeted CRISPR Transcript). The technique consists in the ability of Cas12a endonuclease to cleave non-selective double stranded and single stranded DNA sequences 
triggered when crRNA binds to its complementary target DNA, allowing cleavage of neighboring nucleic acids. In this experiment, DETECTR utilizes collateral cleavage through a reporter nucleic acid that consists of a single stranded DNA containing a fluorophore, so that fluorescence emission is activated in the degradation of target DNA. Finally, another promising system developed called HOLMES (one-Hour Low-cost Multipurpose Highly Efficient System) is an accelerated detection platform for target DNA through a ternary complex (Cas12 - crRNA - target DNA), triggering trans-cleavage of undirected DNA, generating fluorescence lighting. Besides being a diagnostic tool for human disease, HOLMES can also be applied to determine homozygous and heterozygous genotypes, environmental and food monitoring (Figure 2) (Li et al., 2018).

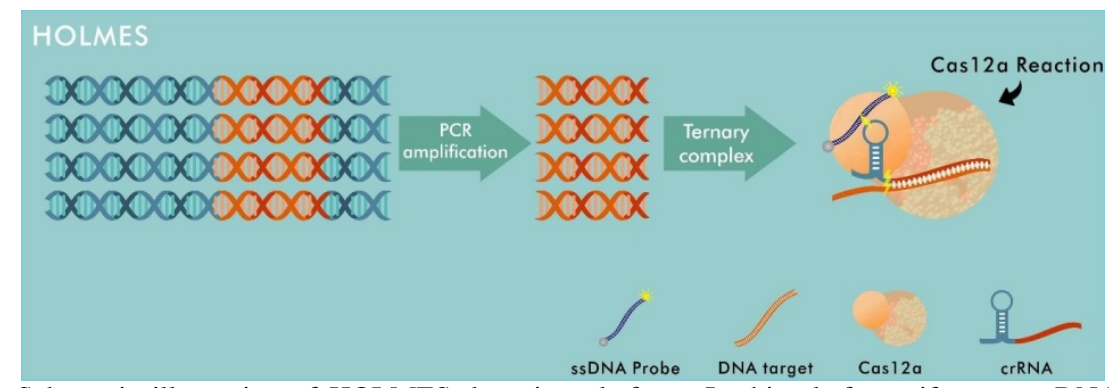

Figure 2. Schematic illustration of HOLMES detection platform. In this platform, if a target DNA is in the reaction system, the Cas $12 /$ crRNA binary complex forms a ternary complex with the target DNA, triggering the Cas 12 trans cleavage of the non-target DNA, generating fluorescence in the system.

\section{Type VI: CRISPR / Cas13}

While most CRISPR / Cas systems target double stranded DNA, type VI systems are known to exclusively target RNA molecules. The Cas13 endonuclease adopts a bilobulated architecture consisting of two distinct ribonucleases. An RNAse (REC) is responsible for pre-crRNA processing to generate mature crRNA and remains in the region after cleavage, forming the surveillance complex with Cas13. While the other RNase activity (NUC) is formed by two HEPN domains (Higher eukaryote-prokaryote nucleotidebinding domain), and together they form the target RNA cleavage site during viral interference step (Ahmadzadeh et al., 2019).

Like Cas12a, Cas13 molecules exhibit activity referred to as "collateral cleavage" when bound to a complementary target RNA (also known as "activator RNA"). The HEPN region becomes able to cut not only the target RNA, but any exposed RNA molecule, including free non-complementary RNA or unbound complementary target RNA, resulting in target degradation as well as cleavage of other RNA sequences (Gootenberg et al., 2018).

Therefore, a great effort is being made to establish and implement new systems capable of recognizing and/or cleaving selective CRISPR/Cas13-based RNA molecules, increasing the usefulness of CRISPR-based research tools. In addition, potential applications for new Cas13-based nucleic acid diagnostic platforms is enormous, including standardized testing for various infectious diseases, leading to more fast and precise clinical decision and point-of-care patient treatments.

The first of these platforms developed for molecular detection comprises the use of Cas13a nuclease "collateral activity" and is called SHERLOCK (High Sensitivity Enzyme 
Blocker for Reporter). This system is based on the combination of Recombinase Polymerase Amplification (RPA) and reverse transcription RPA (RT-RPA) isothermal amplification steps. Following recognition of the target RNA by crRNA-Cas13a complex, a fluorescent reporter RNA is cleaved through collateral activity providing an increase in fluorescent signal resulting in rapid, sensitive and accurate detection of specific RNAs (Gootenberg et al., 2017). More recently Gootenberg et al. (2018) introduced the SHERLOCKv2 platform with significant advantages over the first version. This include quantitative detection approach, enhanced sensitivity (below zeptomolar level), portability and multiplex detection (up to four targets) in single reaction with the use of Cas13, Cas12a and Csm6 proteins (which is a CRISPR type-III effector nuclease activated by cyclic adenylate molecules or linear adenine homopolymers terminated with a $2^{\prime}, 3^{\prime}$-cyclic phosphate). In a further advance, Myhrvold et al. (2018) developed HUDSON (Heating Unextracted Diagnostic Samples to Obliterate Nucleases), a protocol which eliminates the need for nucleic acid extraction and allows the detection of pathogens directly from body fluids. HUDSON linked to SHERLOCK enabled highly sensitive detection of dengue virus in patients' blood, serum and saliva samples in less than two hours (Figure 3A-B).

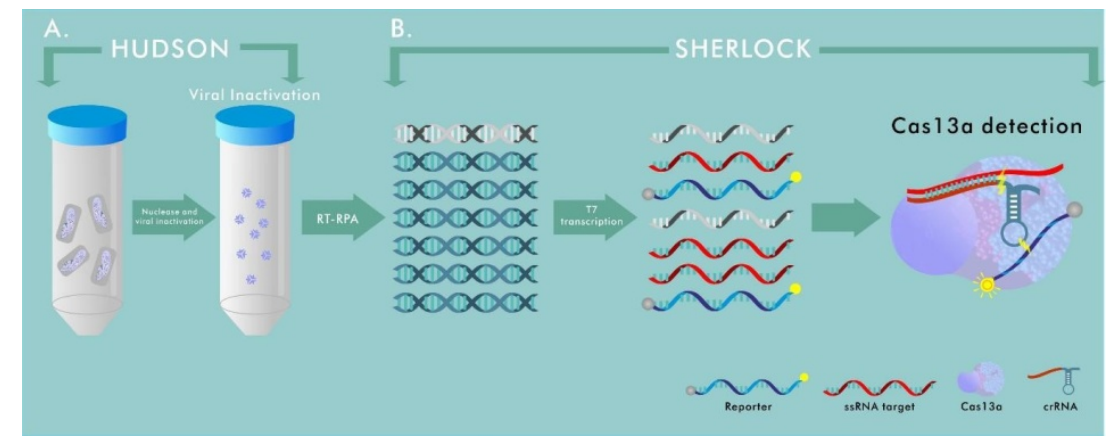

Figure 3. Schematic illustration of rapid diagnostic test associating HUDSON and SHERLOCK. (A) HUDSON is a SHERLOCK-linked method that eliminates the need for nucleic acid extraction through chemical reduction and heat to lyse viral particles and inactivate high levels of nucleases present in body fluids by releasing nucleic acids directly. (B) SHERLOCK integrates RT-RPA isothermal nucleic acid amplification with T7dependent transcription of the target RNA through Cas13-mediated cleavage of nonspecific RNA coupled to a fluorophore.

Finally, Cas13 systems have already been used for other research tools involving target RNA degradation (Abudayyeh et al., 2016), RNA editing (Cox et al., 2017), live cell transcript screening (Abudayyeh et al., 2017), regulation of pre-mRNA splicing (Konermann et al., 2018) and resistance to plant virus RNA (Aman et al., 2018).

\section{FINAL CONSIDERATIONS}

There is no doubt that CRISPR/Cas-based technology, especially using class 2 has provided more accessible and adaptable tools for editing, regulating, visualizing and detecting genomes of different organisms. In addition to enabling rapid development in biological research and biotechnology applications in different areas, the discovery of this prokaryotic immune system has been a milestone in the history of microbiology. Using recent approaches to bioinformatics, a surprising number of new CRISPR/Cas systems have been unveiled, further expanding knowledge about the CRISPR "universe", which will 
serve not only to extend the CRISPR/Cas "toolbox" but also in understanding the various ways which prokaryotes can protect themselves from exogenous nucleic acids. However, as CRISPR revolution continues, several challenges and concerns associated with this technology are also increasing, raising social and ethical issues discussed by scientists and the general population, especially in gene therapy applications. In humans for example, it was recently reported by a Chinese scientist, Dr. He Jiankui, the birth of twins from embryos whose genome had been modified before their transfer to the womb. The modification was performed using CRISPR/Cas9 disabling copies of the CCR gene with the stated goal of preventing transmission of any HIV infection transmitted by the parent. Although a plausible justification appears, the lack of any confirmed assessment of consequences of this action casts doubt on why the scientist performed such a procedure, further raising concerns about these applications, including probable genetic inequality and its long-term ramifications. In addition, concerns such as off-target effect, adaptive immune responses in humans, and inadvertent changes in human germline have been reported. Therefore, CRISPR-based therapy still has a long way to go, with several limitations to address.

Concluding, the perspectives for CRISPR technology are certainly positive. Improvements in conventional tools are expected, and even more spectacular applications are anticipated. Similarly, regulatory agencies will need to consider the best way to promote responsible use of this method without impeding research and technological development.

\section{ACKNOWLEDGMENTS}

We are grateful for research support by FAPEAM (Fundação de Amparo à Pesquisa do Estado do Amazonas).

\section{FUNDING}

Fiocruz Innovation Promotion Program (Inova Fiocruz)

\section{CONFLICTS OF INTEREST}

The authors declare no conflict of interest.

\section{REFERENCES}

Abudayyeh O, Gootenberg JS, Konermann S, Joung J, et al. (2016). C2c2 is a singlecomponent programmable RNAguided RNA-targeting CRISPR effector. Science. 353: aaf5573.

Abudayyeh O, Gootenberg JS, Essletzbichler P, Han S, et al. (2017). RNA targeting with CRISPR-Cas13. Nature. 550: 280-284.

Ahmadzadeh V, Farajnia S, Rahbarnia L, Zarredar H, et al. (2019). CRISPR-Cas system: Toward a more efficient technology for genome editing and beyond. J. Cell Biochem. 19: 114.

Aman R, Ali1 Z, Butt H, Mahas A, et al. (2018). RNA virus interference via CRISPR/Cas13a system in plants. Genome Biol. 19: 1-4.

Barrangou R, Fremaux C, Deveau H, Richards M, et al. (2007). CRISPR Provides Acquired Resistance Against Viruses in Prokaryotes. Science. 315: 1709-1712.

Bikard D, Euler CW, Jiang W, Nussenzweig PM, et al. (2014). Exploiting CRISPR-Cas nucleases to produce sequencespecific antimicrobials. Nat. Biotechnol. 32: 1146-1150.

Bolotin A, Quinquis B, Sorokin A, Ehrlich SD, et al. (2005). Clustered regularly interspaced short palindrome repeats (CRISPRs) have spacers of extrachromosomal origin. Microbiology. 151: 2551-2561. 
Chandrasekaran J, Brumin M, Wolf D, Leibman D, et al. (2016). Development of broad virus resistance in nontransgenic cucumber using CRISPR/Cas9 technology. Mol. Plant Pathol. 17: 1140-1153.

Chen JS, Ma E, Harrington LB, Da Costa M, et al. (2018). CRISPR-Cas12a target binding unleashes indiscriminate single-stranded DNase activity. Science. 360: 436-439.

Cox DBT, Gootenberg JS, Abudayyeh O, Franklin B, et al. (2017). RNA editing with CRISPR-Cas13. Science. 358 : 1019-1027.

Deltcheva E, Chylinski K, Sharma CM, Gonzales K, et al. (2011). CRISPR RNA maturation by trans-encoded small RNA and host factor RNase III. Nature. 471: 602-607.

Du QS, Cui J, Zhang CJ, He K, et al. (2016). Visualization analysis of CRISPR/Cas9 gene editing technology studies. $J$. Zhejiang Univ. Sci. 17: 798-806.

Egorova TV, Zotova ED, Reshetov DA, Polikarpova AV, et al. (2019). CRISPR/Cas9generated mouse model of Duchenne muscular dystrophy recapitulating a newly identified large $430 \mathrm{~kb}$ deletion in the human DMD gene. Dis. Model. Mech. 12: dmm037655.

Garneau JE, Dupuis ME, Villion M, Romero DA, et al. (2010). The CRISPR/Cas bacterial immune system cleaves bacteriophage and plasmid DNA. Nature. 468: 67-71.

Gootenberg JS, Abudayyeh OO, Lee JW, Essletzbichler P, et al. (2017). Nucleic acid detection with CRISPRCas13a/C2c2. Science. 356: 438-442.

Gootenberg JS, Abudayyeh OO, Kellner MJ, Joung J, et al. (2018). Multiplexed and portable nucleic acid detection platform with Cas13, Cas12a, and Csm6. Science. 360: 439-444.

Ishino Y, Shinagawa H, Makino K, Amemura M, et al. (1987). Nucleotide sequence of the iap gene, responsible for alkaline phosphatase isoenzyme conversion in Escherichia coli, and identification of the gene product. J. Bacteriol. 169: 5429-5433

Jansen R, Embden JD, Gaastra W, Schouls LM, et al. (2002). Identification of genes that are associated with DNA repeats in prokaryotes. Mol. Microbiol. 43: 1565-1575.

Jeon Y, Choi YH, Jang Y, Yu J, et al. (2018). Direct observation of DNA target searching and cleavage by CRISPRCas12a. Nat. Commun. 9: 2777.

Jinek M, Chylinski K, Fonfara I, Hauer M, et al. (2012). A programmable dual-RNAguided DNA endonuclease in adaptive bacterial immunity. Science. 337: 816-821.

Kaminski R, Chen Y, Fischer T, Tedaldi E, et al. (2016). Elimination of HIV-1 Genomes from Human T-lymphoid Cells by CRISPR/Cas9 Gene Editing. Sci. Rep. 6: 22555.

Kaur N, Alok AS, Kaur N, Pandey P, et al. (2018). CRISPR/Cas9-mediated efficient editing in phytoene desaturase (PDS) demonstrates precise manipulation in banana cv. Rasthali genome. Funct. Integr. Genomics. 18: 89-99.

Konermann S, Lotfy P, Brideau NJ, Oki J, et al. (2018). Transcriptome Engineering with RNA-Targeting Type VI-D CRISPR Effectors. Cell. 173: 665- 676.

Koonin EV, Makarova KS and Zhang F (2017). Diversity, classification and evolution of CRISPR-Cas systems. Curr. Opin. Microbiol. 37: 67-78.

Li C, Ding L, Sun CW, Wu LC, et al. (2016). Novel HDAd/EBV Reprogramming Vector and Highly Efficient Ad/CRISPR-Cas Sickle Cell Disease Gene Correction. Sci. Rep. 6: 30422.

Li Qi, Chen J, Minton NP, Zhang Y, et al. (2016). CRISPR-based genome editing and expression control systems in Clostridium acetobutylicum and Clostridium beijerinckii. Biotechnol. J. 11: 961-972.

Li SY, Cheng QX, Wang JM, Li XY, et al. (2018). CRISPR-Cas12a-assisted nucleic acid detection. Cell Discov. 4: 20.

Makarova KS, Wolf YI, Alkhnbashi OS, Costa F, et al. (2015). An updated evolutionary classification of CRISPR-Cas systems. Nat. Ver. Microbiol. 13: 722-736.

Marraffini LA and Sontheimer EJ (2010). Self versus non-self discrimination during CRISPR RNA-directed immunity. Nature. 463: 568-571.

Mohanraju P, Makarova KS, Zetsche B, Zhang F, et al. (2016). Diverse evolutionary roots and mechanistic variations of the CRISPR-Cas systems. Science. 353: aad5147.

Mojica FJM, Diez-Villasenor C, García-Martınez J and Soria E (2005). Intervening Sequences of Regularly Spaced Prokaryotic Repeats Derive from Foreign Genetic Elements. J. Mol. Evol. 60: 174-182.

Mojica FJM and Rodriguez-valera F (2016). The discovery of CRISPR in archaea and bacteria. FEBS J. 283: 31623169.

Myhrvold C, Freije CC, Gootenberg JS, Abudayyeh O, et al. (2018). Field-deployable viral diagnostics using CRISPRCas13. Science. 360: 444-448.

Nishimasu H, Ran FA, Hsu PD, Konermann S, et al. (2014). Crystal structure of Cas9 in complex with guide RNA and target DNA. Cell. 156: 935-949.

Nishitani C, Hirai N, Komori S, Wada M, et al. (2016). Efficient Genome Editing in Apple Using a CRISPR/Cas9 system. Sci. Rep. 6: 31481.

Pourcel C, Salvignol G and Vergnaud G (2005). CRISPR elements in Yersinia pestis acquire new repeats by preferential uptake of bacteriophage DNA, and provide additional tools for evolutionary studies. Microbiology. 151: 653-663.

Ren J and Zhao Y (2017). Advancing chimeric antigen receptor T cell therapy with CRISPR/Cas9. Protein Cell. 8: 634643. 
Safari F, Zare K, Negahdaripour M, Barekati-Mowahed M, et al. (2019). CRISPR Cpf1 proteins: structure, function and implications for genome editing. Cell Biosci. 9: 36.

Schwank G, Koo BK, Sasselli V, Dekkers JF, et al. (2013). Functional repair of CFTR by CRISPR/Cas9 in intestinal stem cell organoids of cystic fibrosis patients. Cell Stem Cell. 13: 653-658.

Shi J, Gao H, Wang H, Lafitte HR, et al. (2017). ARGOS8 variants generated by CRISPRCas9 improve maize grain yield under field drought stress conditions. Plant Biotechnol. J. 15: 207-216.

Shmakov S, Abudayyeh OO, Makarova KS, Wolf YI, et al. (2015). Discovery and Functional Characterization of Diverse Class 2 CRISPR-Cas Systems. Mol. Cell. 60: 385- 397.

Teng F, Li J, Cui T, Xu K, et al. (2019). Enhanced mammalian genome editing by new Cas12a orthologs with optimized crRNA scaffolds. Genome Biol. 20: 15.

Tsukamoto T, Sakai E, Iizuka S, Taracena-Gándara M, et al. (2018). Generation of the Adenovirus Vector-Mediated CRISPR/Cpf1 System and the Application for Primary Human Hepatocytes Prepared from Humanized Mice with Chimeric Liver. Biol. Pharm. Bull. 41: 1089-1095.

Van Der Oost J, Jore MM, Westra ER, Lundgren M, et al. (2009). CRISPR-based adaptive and heritable immunity in prokaryotes. Trends Biochem. Sci. 34: 401-407.

Van Soolingen D, Haas PE, Hermans PW, Groenen PM, et al. (1993). Comparison of various repetitive DNA elements as genetic markers for strain differentiation and epidemiology of Mycobacterium tuberculosis. J. Clin. Microbiol. 31: 1987-1995.

Wang X, Huang X, Fang X, Zhang Y, et al. (2016). CRISPR-Cas9 System as a Versatile Tool for Genome Engineering in Human Cells. Mol. Ther. Nucleic Acids. 5: e388.

Wang Z, Wang S, Li D, Zhang Q, et al. (2018). Optimized paired-sgRNA/Cas9 cloning and expression cassette triggers high-efficiency multiplex genome editing in kiwifruit. Plant Biotechnol. J. 16: 1424-1433.

Wang Z, Wang W, Cui YC, Pan Q, et al. (2018). HIV-1 Employs Multiple Mechanisms To Resist Cas9/Single Guide RNA Targeting the Viral Primer Binding Site. J. Virol. 92: e01135-18.

Wiedenheft B, Lander GC, Zhou K, Jore MM, et al. (2011). Structures of the RNA-guided surveillance complex from a bacterial immune system. Nature. 477: 486-489.

Wu H, Liu Q, Shi H, Xie J, et al. (2018). Engineering CRISPR/Cpf1 with tRNA promotes genome editing capability in mammalian systems. Cell Mol. Life Sci. 75: 3593-3607.

Yosef I, Goren MG and Qimron U (2012). Proteins and DNA elements essential for the CRISPR adaptation process in Escherichia coli. Nucleic Acids Res. 40: 5569-5576.

Zeisel MB, Lucifora J, Mason WS, Sureau C, et al. (2015). Towards an HBV cure: state-of-the-art and unresolved questions--report of the ANRS workshop on HBV cure. Gut. 64: 1314-1326.

Zhang X, Wang J, Cheng Q, Zheng X, et al. (2017). Multiplex gene regulation by CRISPR-ddCpf1. Cell Discov. 3: 17018.

Zhao Z, Shi L, Zhang W, Han J, et al. (2018). CRISPR knock out of programmed cell death protein 1 enhances antitumor activity of cytotoxic T lymphocytes. Oncotarget. 9: 5208-5215.

Zhen S, Lu J, Wang LJ, Sun XM, et al. (2016). In Vitro and In Vivo Synergistic Therapeutic Effect of Cisplatin with Human Papillomavirus16 E6/E7 CRISPR/Cas9 on Cervical Cancer Cell Line. Transl. Oncol. 9: 498-504.

Zhou J, Wang G and Liu Z (2018). Efficient genome editing of wild strawberry genes, vector development and validation. Plant Biotechnol. J. 16: 1868-1877. 